\title{
CRISPR/Cas14a-based Isothermal Amplification for Profiling Plant
}

\section{MicroRNAs}

Hao Yang ${ }^{\mathrm{a}}$, Junbo Chen ${ }^{\mathrm{b}}$, Sen Yang ${ }^{\mathrm{a}}$, Ting Zhang ${ }^{\mathrm{a}}$, Xuhan Xia ${ }^{\mathrm{a}}$, Kaixiang Zhang ${ }^{\mathrm{c}}$, Sha Deng ${ }^{a}$, Guiping He ${ }^{a}$, Hong Gao ${ }^{a}$, Qiang He ${ }^{a}$, Ruijie Denga,*

a College of Biomass Science and Engineering, Healthy Food Evaluation Research Center, Sichuan University, Chengdu 610065, China

${ }^{b}$ Analytical \& Testing Center, Sichuan University, Chengdu, Sichuan 610064, China

${ }^{c}$ School of Pharmaceutical Sciences, Key Laboratory of Targeting Therapy and Diagnosis for Critical Diseases, Zhengzhou University, Zhengzhou 450001, China

* Corresponding author:

e-mail: drj17@scu.edu.cn

Address: College of Biomass Science and Engineering, Healthy Food Evaluation Research Center, Sichuan University, Chengdu 610065, China; Tel: +86 02885467382. 


\section{Table of content}

Table S1. Oligonucleotide sequences

Figure S1. The test of the Cas14R assay for detecting miR172

Figure S2. The opimization of the ligation time of the Cas14R assay for miR156a detection .85

Figure S3. Standard color chart for banana ripeness .56

Figure S4. Fluorescence spectra of the Cas14R assay towards miR156a extracted from various banana ripeness (stage2, 4 and 7 ).

Figure S5. RT-qPCR curves for measuring miR156a extracted from various banana ripeness (stage2, 4 and 7 ). .58

Figure S6. Profiling miR156a in arabidopsis thaliana and maize and salmonella typhimurium. S9

Figure S7. Plasmid vector for constructing Cas14a-sgRNA-T. $S 11$ 
Table S1. Oligonucleotide sequences

\begin{tabular}{|c|c|}
\hline Oligonucleotides & Sequences ( $5^{\prime}$ to $3^{\prime}$ ) \\
\hline miR156a & UGACAGAAGAGAGUGAGCAC - 2' me \\
\hline $\operatorname{miR} 156 e$ & UGACAGAAGAUAGAGAGCAC - 2 ' me \\
\hline miR156n & UGACAGGAGAGAGUGAGCAC - $2^{\prime}$ me \\
\hline $\operatorname{miR} 156 \mathrm{~g}$ & GCUCACUGCUCUCUCUGUCAUC - 2 ' me \\
\hline $\operatorname{miR} 156 p$ & GCUCAUUUCUCUUUCUGUCAGC - 2 'me \\
\hline $\operatorname{miR} 172$ & AGAAUCUUGAUGAUGCUGCAU \\
\hline \multirow{2}{*}{ Padlock probe } & TCTTCTGTCACATCCTGATAAAGAACAGCAACCGATAAGATAAGTTAAGG \\
\hline & TGCTCACTC \\
\hline \multirow[t]{3}{*}{ primer } & CTTAACTTATCTTATCGGTTG \\
\hline & CUUCACUGAUAAAGUGGAGAACCGCUUCACCAAAAGCUGUCCCUUAGGGG \\
\hline & AUUAGAACUUGAGUGAAGGUGGGCUGCUUGCAUCAGCCUAAUGUCGAGAA \\
\hline \multirow[t]{5}{*}{ Cas14a-sgRNA } & GUGCUUUCUUCGGAAAGUAACCCUCGAAACAAAUUCAUUUUUCCUCUCCA \\
\hline & AUUCUGCACAAGAAAGUUGCAGAACCCGAAUAGACGAAUGAAGGAAUGCA \\
\hline & ACCAUCCUGAUAAAGAACAGCAACC \\
\hline & CAAATACGACGTGTCAACGGTAATACGACTCACTATAGGGCTTCACTGAT \\
\hline & AAAGTGGAGAACCGCTTCACCAAAAGCTGTCCCTTAGGGGATTAGAACTT \\
\hline \multirow{4}{*}{ Cas14a-sgRNA-T } & GAGTGAAGGTGGGCTGCTTGCATCAGCCTAATGTCGAGAAGTGCTTTCTT \\
\hline & CGGAAAGTAACCCTCGAAACAAATTCATTTTTCСTCTCCAATTCTGCACA \\
\hline & AGAAAGTTGCAGAACCCGAATAGACGAATGAAGGAATGCAACCATCCTGA \\
\hline & TAAAGAACAGCAACCTATATA \\
\hline sgRNA-fwd & CAAATACGACGTGTCAACGG \\
\hline sgRNA-rev & GGTTGCTGTTCTTTATCAGGATG \\
\hline FQ-12T & FAM-TTTTTTTTTTTT - BHQ1 \\
\hline RT probe & GTCGTATCCAGTGCAGGGTCCGAGGTATTCGCACTGGATACGACGTGCTC \\
\hline RT-qPCR-F & GCCGCTGACAGAAGAGAGTG \\
\hline RT-qPCR-R & GTGCAGGGTCCGAGGT \\
\hline
\end{tabular}

*The bold parts represent the targeting regions of the Cas14a-sgRNA. The underlined region are the reverse complementary sequences to sgRNA-fwd and sgRNA-rev respectively. The italic part is the $\mathrm{T} 7$ promoter sequence. 


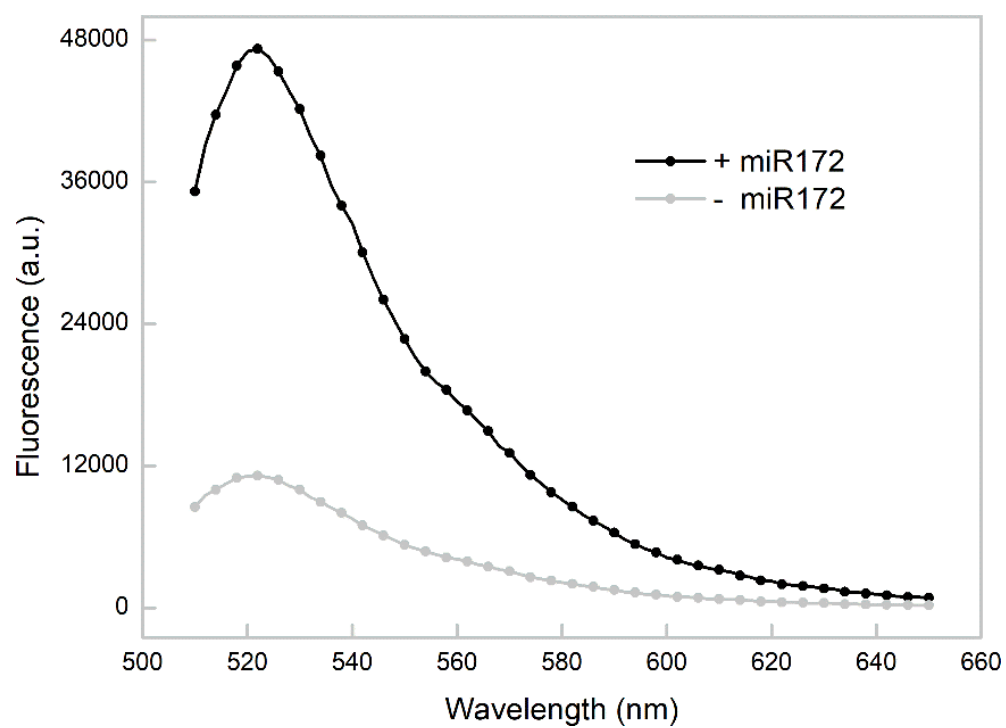

Figure S1. The test of the Cas14R assay for detecting miR172. The final concentrations of phosphorylated padlock probe, primer, Cas14a-sgRNA, miR172, FQ-12T, T4 DNA Ligase, phi29 DNA polymerase and Cas14a protein were $10 \mathrm{nM}, 10 \mathrm{nM}, 100 \mathrm{nM}, 10 \mathrm{nM}, 400 \mathrm{nM}$ and 0.0625 Weiss $\mathrm{U} / \mu \mathrm{L}, 0.075 \mathrm{U} / \mu \mathrm{L}$ and $50 \mathrm{nM}$, respectively. 


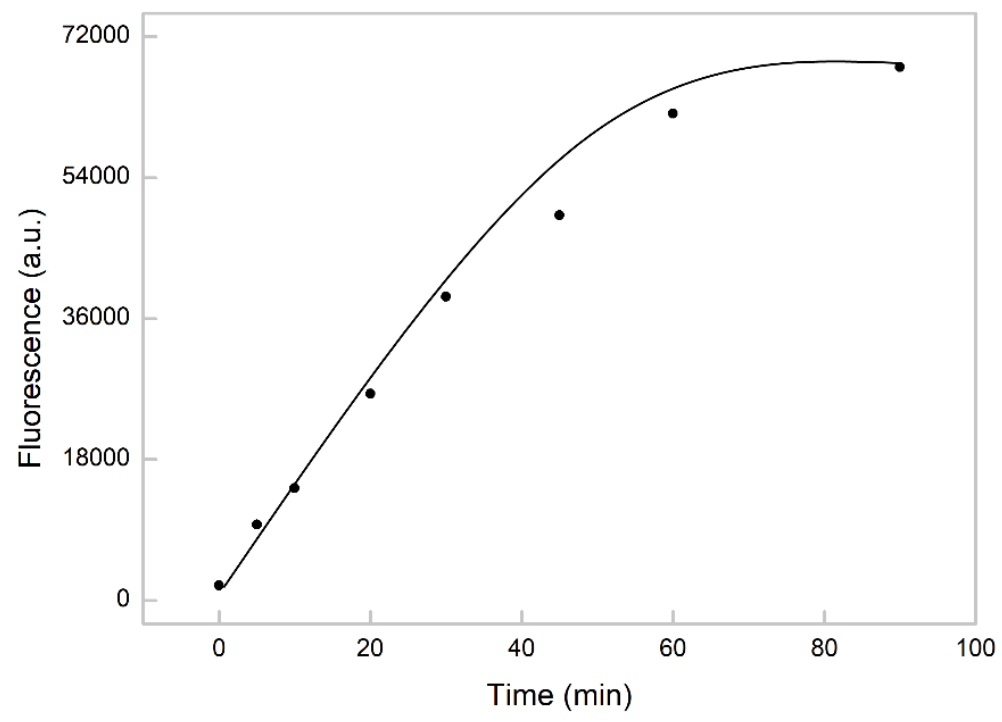

Figure S2. The opimization of the ligation time of the Cas14R assay for miR156a detection.

The excitation wavelength was $480 \mathrm{~nm}$, and the emission wavelength was $520 \mathrm{~nm}$. The final concentrations of phosphorylated padlock probe, primer, Cas14a-sgRNA, activator, FQ-12T, T4 DNA Ligase, phi29 DNA polymerase and Cas14a protein were $10 \mathrm{nM}, 10 \mathrm{nM}$, $100 \mathrm{nM}, 100 \mathrm{nM}, 400 \mathrm{nM}$ and 0.0625 Weiss $\mathrm{U} / \mu \mathrm{L}, 0.075 \mathrm{U} / \mu \mathrm{L}$ and $50 \mathrm{nM}$, respectively. 


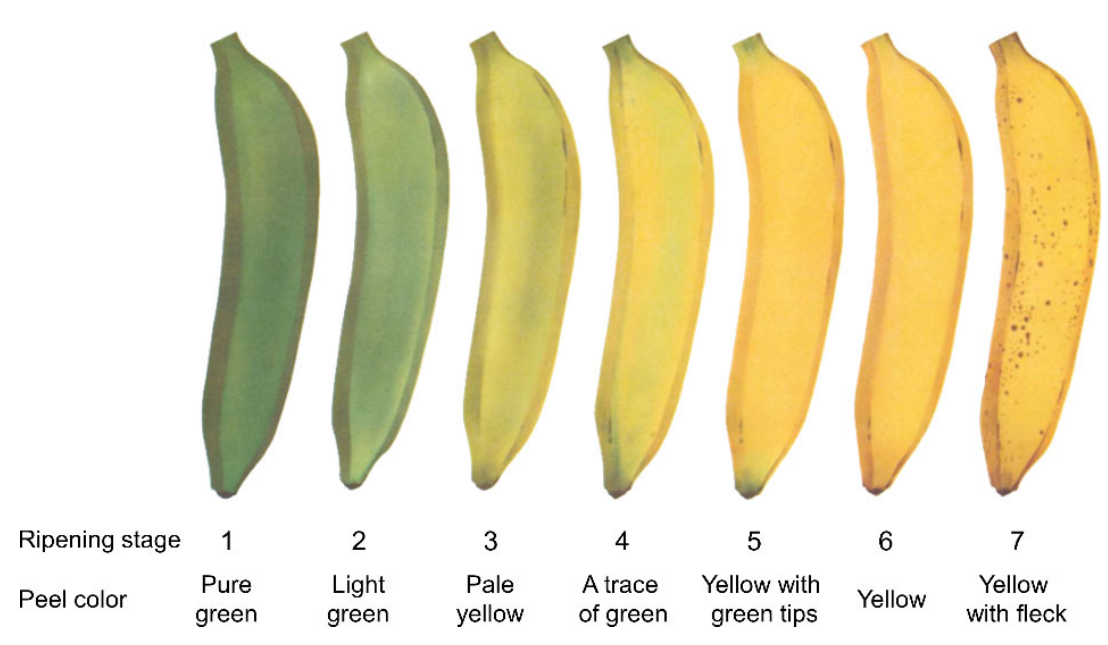

Figure S3. Standard color chart for banana ripeness. 


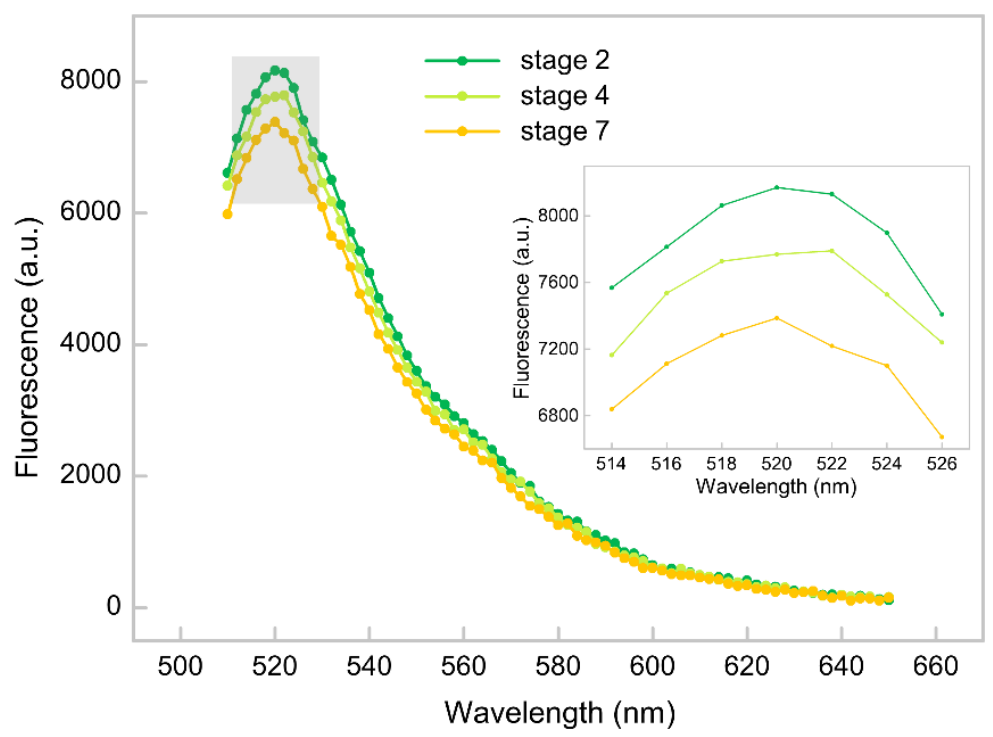

Figure S4. Fluorescence spectra of the Cas14R assay towards miR156a extracted from various banana ripeness (stage2, 4 and 7). 


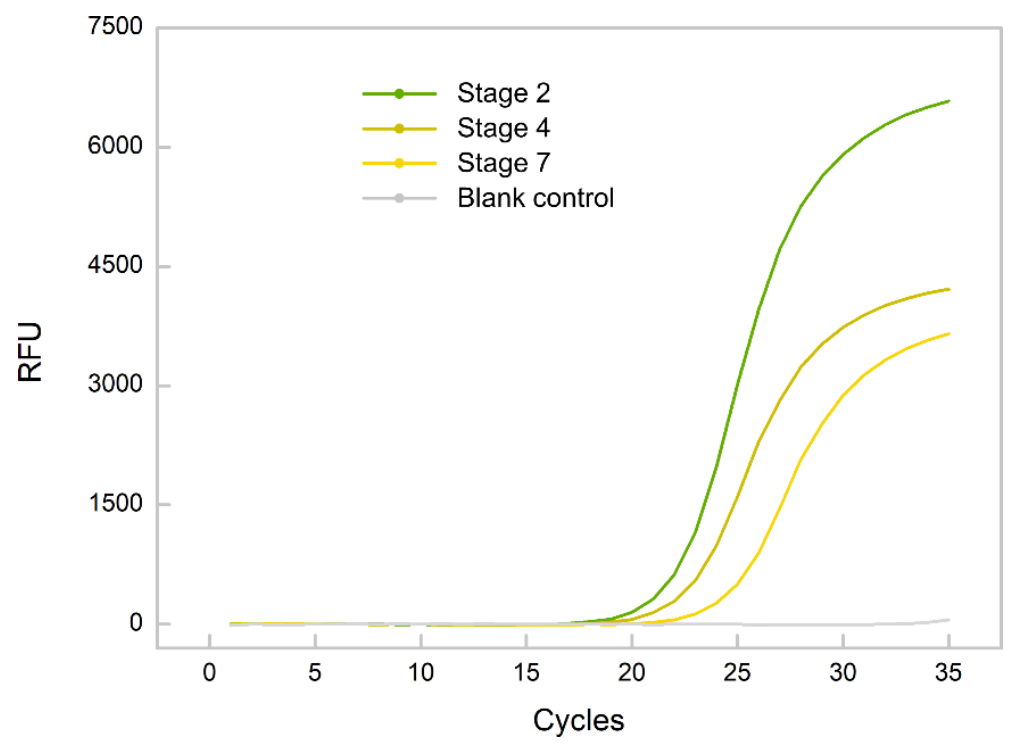

Figure S5 RT-qPCR curves for measuring miR156a extracted from various banana ripeness (stage2, 4 and 7). 

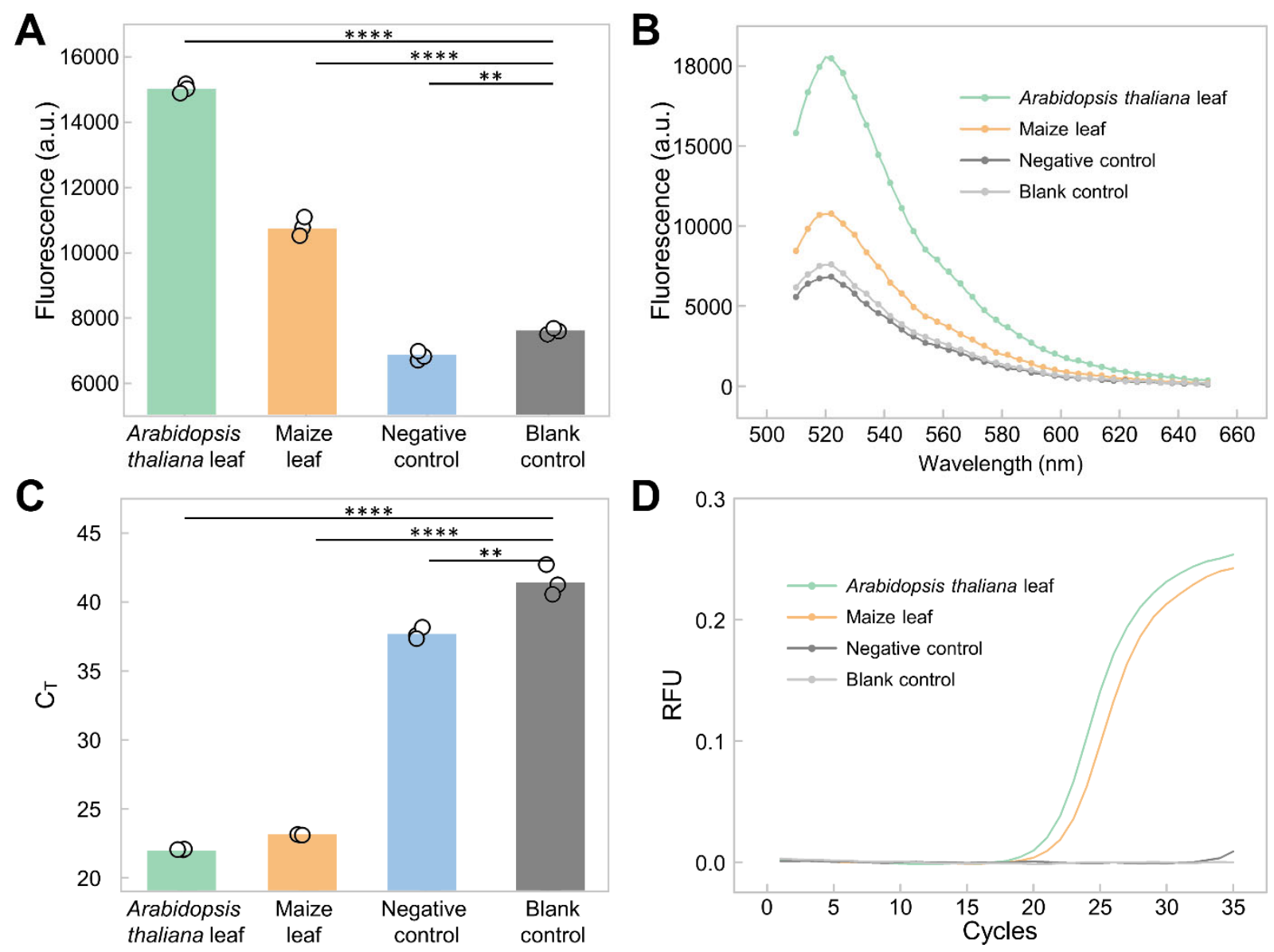

Figure S6. Profiling miR156a in arabidopsis thaliana and maize and salmonella typhimurium. (A) Histogram of fluorescence response of the Cas $14 R$ assay towards miR156a extracted from arabidopsis thaliana leaf, maize leaf, salmonella typhimurium (negative control) and $\mathrm{H}_{2} \mathrm{O}$ (blank control). (B) Spectra of fluorescence response of the Cas14R assay towards miR156a extracted from arabidopsis thaliana leaf, maize leaf, salmonella typhimurium (negative control) and $\mathrm{H}_{2} \mathrm{O}$ (blank control). (C) Histogram of RT-qPCR results for measuring miR156a extracted from arabidopsis thaliana leaf, maize leaf, salmonella typhimurium (negative control) and $\mathrm{H}_{2} \mathrm{O}$ (blank control). (D) RT-qPCR curves for measuring miR156a extracted from arabidopsis thaliana leaf, maize leaf, salmonella typhimurium (negative control) and $\mathrm{H}_{2} \mathrm{O}$ (blank control). Tests were carried out 
by 3 parallel measurements. Statistical significance data were obtained by two-tailed unpaired Student's t-test: ${ }^{* * * *} \mathrm{P}<0.0001$. 


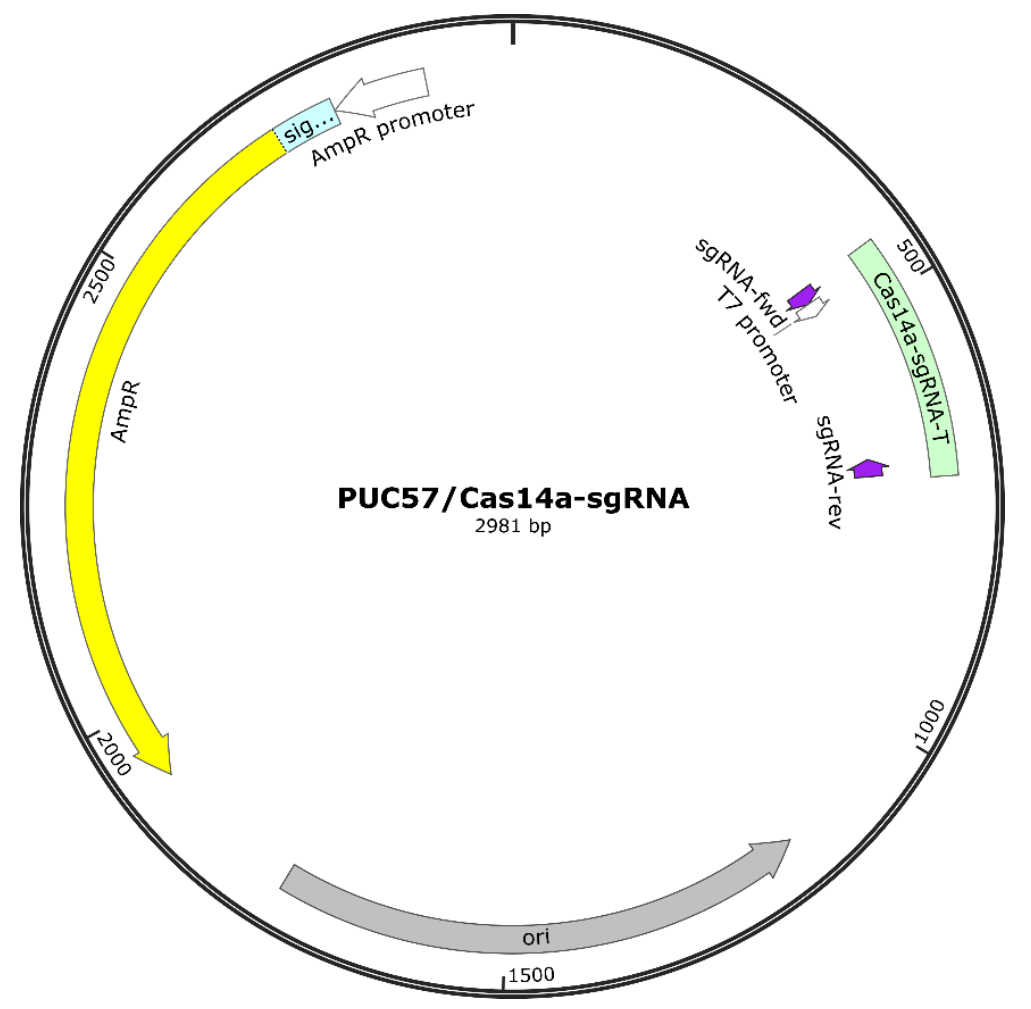

Figure S7. Plasmid vector for constructing Cas14a-sgRNA-T. 